\title{
Venipuncture in bats
}

\author{
David Eshar, DVM 1 \& Maya Weinberg, DVM²
}

\section{Though not as common as small rodents in laboratory settings, bats are being increasingly used in research studies. Knowledge of proper blood sampling techniques is essential for care and management of bats.}

In recent years, interest in research using bats has grown. These small mammals have many unique features that attract great attention and can now be more commonly found in many research centers ${ }^{1}$. Second to rodents, bats are the most abundant and diverse mammalian group in nature. The order Chiroptera is divided into suborders Microchiroptera and Megachiroptera, with further subdivision into 18 families and 1,116 recognized species.

Bats used in studies are either wildcaught or originate from captive breeding colonies $^{1}$. Blood testing in bats may be done as part of a clinical evaluation or for hematological research.

Sites for blood collection from bats include the median (brachial) vein, cranial vena cava, jugular vein, orbital sinus and heart ${ }^{2-4}$. Venipuncture of the cephalic and the saphenous (interfemoral) veins are two of the best techniques for quick and safe collection of blood in bats. These nonterminal techniques can be used to collect small to medium volumes of blood with good visualization of the blood vessels and can be done on anesthetized or manually restrained, unsedated bats.

\section{Anatomy and physiology}

The cephalic vein is located along the leading edge of the patagium or the antebrachial wing membrane (Fig. 1). The saphenous (interfemoral) vein is found in the uropatagium (tail membrane) parallel to the femur (Fig. 2).

The blood volume of bats comprises $\sim 10 \%$ of the total body weight $(9.0-11.0$ $\mathrm{ml}$ per $100 \mathrm{~g})^{2}$. As in other species, it is considered safe to remove a blood sample equivalent to $1 \%$ of the total body weight at each draw.

\section{Restraint}

Several bat species can act as reservoir hosts of zoonotic pathogens including Lyssaviruses, severe acute respiratory syndrome (SARS) coronavirus, Ebola virus, Henipaviruses, West Nile virus, St. Louis encephalitis virus and Leptospira spp. ${ }^{2,5}$. All personnel working with bats should be vaccinated against rabies $^{2}$. Coats and gloves should be used to minimize contact with body secretions, such as blood or saliva, that may contain infectious organisms $^{2}$.

Manual restraint is usually not a problem in bats, but healthy bats can wriggle excessively unless properly restrained. All restrained bats can deliver a defensive bite, and the digits and associated claws of large bats can scratch an unprotected handler or pull fingers in for a bite ${ }^{2}$. Bats should be restrained using either protective thick gloves or a towel to hold the head and the limbs (Fig. 3). The duration of manual restraint should be minimized to reduce stress and prevent hyperthermia and marked elevations in cortisol and glucose ${ }^{6}$.

Stressed bats may require chemical restraint, either by inhalation or by injection, although chemical restraint may have complicating side effects. Bats should be anesthetized for procedures requiring close contact to reduce risk of biting and transmission of secretions ${ }^{2}$.

Bats are homeotherms, maintaining their body temperature between $35^{\circ} \mathrm{C}$ and $39^{\circ} \mathrm{C}$ (ref. 2). Additionally, large lungs and naked flight membranes result in greater heat loss (6 times greater) and thermal conductance

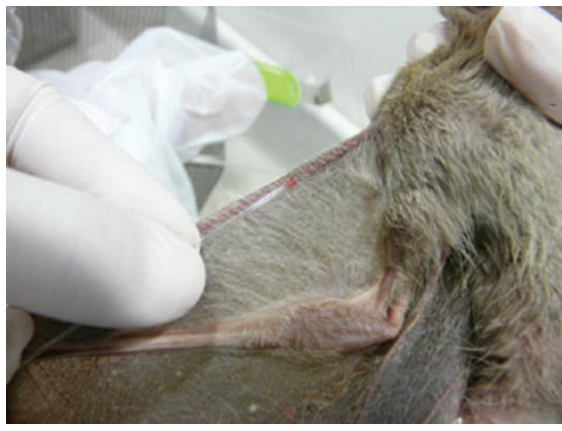

FIGURE 1 | A blood sample is obtained from the cephalic vein in the patagium of an Egyptian fruit bat (Rousettus aegyptiacus) using a microhematocrit tube.

(1.5-4 times greater) than is common in wingless animals ${ }^{2}$. Therefore, bats should be kept in their optimal temperature zone at all times, actively heated when anesthetized; furthermore, the use of alcohol-based scrub solutions can cause further heat loss and should be avoided. Bats use a large amount of energy to compensate for their heat loss and they consume large quantities of food relative to their body size ${ }^{2}$; therefore, restrained animals should be fed shortly before and after the procedure.

\section{Preparation}

To minimize handling and to avoid sample failure, all needed equipment should be prearranged and organized in advance. Equipment needed includes a 1- to 3-ml syringe or $0.5-\mathrm{ml}$ insulin syringe; 25 - or 27-gauge needles; microtainer collection tubes with heparin and calcium-EDTA; microhematocrit capillary tubes and sealing clay; glass slides; chlorhexidine-based scrub 


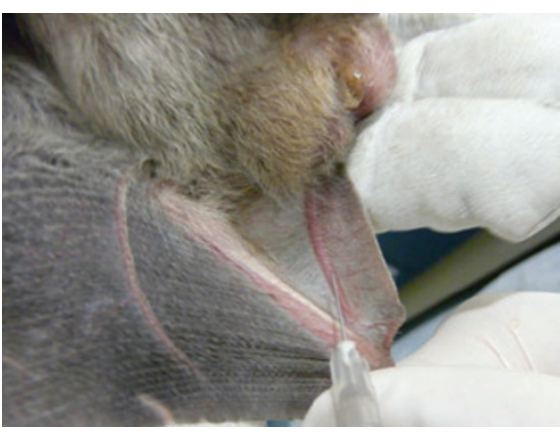

FIGURE 2 | Venipuncture of the right saphenous (interfemoral) vein in the uropatagium of a male Egyptian fruit bat (Rousettus aegyptiacus) using a 1 -ml syringe with a 27 -gauge needle.

solution; leather and non-sterile gloves; and chemical restraint agents (as needed).

Some bat species weigh less than $100 \mathrm{~g}$, and in many cases, only a small sample is obtained. Hence, we prefer to use blood analyzers (such as the Abaxis Vetscan chemistry analyzer) that require only small blood sample volumes $(0.1 \mathrm{ml}$ of whole blood or plasma).

To avoid clotting and to reduce sample collection time, syringes should be prepared with heparin in advance ${ }^{7}$.

\section{Collection methods}

Venipuncture of the cephalic and saphenous veins without anesthesia usually requires one phlebotomist and one or two individuals to restraint the bat. One assistant will hold the bat's head and body while a second assistant gently spreads the wing and holds the vein at its most proximal end, to allow the vessel to engorge with blood and dilate. For a right-handed phlebotomist, the right wing of the bat is extended while the left wing is securely held by the first assistant. The left hand of the phlebotomist is used to either compress the vein or add stability while the right hand holds the syringe.

The venipuncture site can be warmpacked prior to the procedure to facilitate blood flow. Aseptic techniques should be used for the venipuncture using a chlorhexidine-based scrub solution.

For most cases, a 25-gauge needle with a 1 -ml syringe or a $0.5-\mathrm{ml}$ insulin syringe with attached 27-gauge needle is used (Fig. 2). Once the needle is inserted into the vessel and blood is identified in the needle hub, a gentle negative pressure (suction) is intermittently applied until the desired sample volume is obtained. Care should be taken not to use excessive pressures that can cause collapse of the vein or hemolysis of the blood sample.

Alternatively, microhematocrit capillary tubes can be used for sample collection. A 25 -gauge needle can be used to puncture the vessel in small bats, and the tube can be inserted into the hub of the needle to collect the sample, or if a vein is accidently punctured, blood can be collected into a microhematocrit capillary tube directly from the incision site ${ }^{2}$ (Fig. 1).

Bats' wing membranes are very thin and lack subcutaneous fat to aid in hemostasis of the punctured blood vessels. The venipuncture site should be gently pressed using a piece of cotton gauze until bleeding completely stops. Special attention should be given to small bats because large hematomas and ongoing bleeding can result in severe and even life-threatening blood loss. Once back in its cage, the bat should be closely monitored for renewed bleeding resulting from wing flapping.

\section{Sample handling}

Bat blood can altered by inappropriate collection, handling and storage ${ }^{2}$. Moderate hemolysis during sample collection can cause test results to show hyperkalemia, hyponatremia, hypochloremia and elevated levels of aspartate transaminases ${ }^{8}$.

The sample should be processed within 6 hours of sampling, as prolonged contact between the resting plasma and the red cells causes elevation in phosphorus and decreases in chloride and glucose concentrations ${ }^{8}$.

Blood samples treated with heparin can be analyzed immediately after collection and

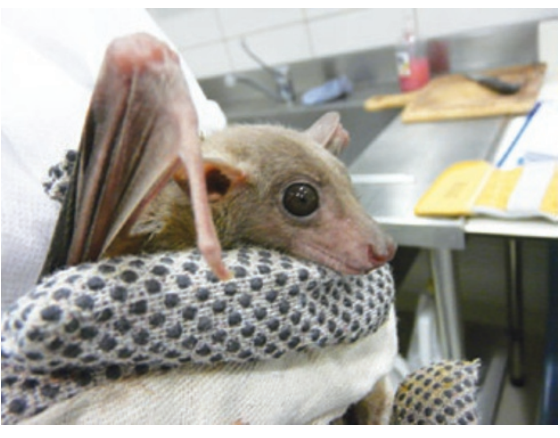

FIGURE 3 | Physical restraint of an Egyptian fruit bat (Rousettus aegyptiacus) for sample collection. The restrainer manually holds the bat's neck and feet using thick gloves to protect against bites.

can also be centrifuged so that plasma can be removed and frozen until later analysis ${ }^{7,8}$.

\section{Conclusion}

Cephalic and saphenous (interfemoral) venipuncture is a non-terminal technique that allows simple, safe and efficient blood sampling in bats of various sizes.

1. Fenton, M.B. Science and the conservation of bats. J. Mammal. 78, 1-14 (1997).

2. Heard, D.J. Chiropterans (bats). in Zoo Animal And Wildlife Immobilization And Anesthesia (eds. West, G., Heard, D.J. \& Caulkett, N.) 359-365 (Blackwell Publishing, Ames, IA, 2007).

3. Baer, G.M. A method of bleeding small bats. J. Mammal. 47, 340 (1966).

4. Baer, G.M. \& McLean, R.G. A new method of bleeding small and infant bats. J. Mammal. 53, 231-232 (1972).

5. Van der Poel, W.H., Lina, P.H. \& Kramps, J.A. Public health awareness of emerging zoonotic viruses of bats: a European perspective. Vector Borne Zoonotic Dis. 6, 315-324 (2006).

6. Widmaier, E.P. \& Kunz, T.H. Basal, diurnal, and stress-induced levels of glucose and glucocorticoids in captive bats. J. Exp. Zool. 265, 533-540 (1993).

7. Heard, D.J., Ruiz, M.M. \& Harr, K.E. Comparison of serum and plasma for determination of blood biochemical values in Malaysian flying foxes (Pteropus vampyrus). J. Zoo Wildl. Med. 37, 245-248 (2006).

8. Day, R.L., Heard, D.J. \& LaBlanc, D. The effect of time at which plasma separation occurs on biochemical values in small island flying foxes (Pteropus hypomelanus). J. Zoo Wildl. Med. 32 206-208 (2001). 